\title{
Study of Corrections to the Geometrical Factor in the Space Charge Impedance for the IPNS Upgrade
}

\author{
K. C. Harkay \\ Argonne National Laboratory
}

\section{Introduction}

Collective instabilities are an important consideration in the $2-\mathrm{GeV}$ rapidly-cycling synchrotron (RCS) of the proposed 1-MW spallation neutron source upgrade due to the very high beam intensity of $1.04 \times 10^{14}$ protons per pulse. Collective instabilities are intensity-dependent effects which arise due to the electromagnetic wake fields generated by the beam as it interacts with its surroundings. The interactions are characterized by the coupling impedance, which in the RCS is dominated by space charge effects. To minimize the space charge impedance, the vacuum chamber is constructed with a special wire rf shield.

Estimating the longitudinal and transverse impedance due to space charge is critical for the beam stability analysis. The standard geometrical factors used to evaluate the space charge impedance assume a uniform, round, unbunched beam in a cylindrical, smooth beam pipe. Two corrections to the geometrical factors have been proposed to account separately for the wire rfscreening cage and the more realistic varying elliptical beam cross-section. 5,6 These corrections are studied in the case of the RCS. It is found that including these details results in a correction of less than $20 \%$.

\section{Standard $g$ factors}

The longitudinal $\left(Z_{11}\right)$ and transverse $\left(Z_{1}\right)$ impedance due to the self-fields of a charged beam in a vacuum chamber, commonly called the space charge impedance, is given by $1-3$

$$
\frac{Z_{11}}{n}=-j \frac{Z_{0}}{2 \beta \gamma^{2}} g_{0} \quad Z_{\perp}=-j \frac{R Z_{0}}{\beta^{2} \gamma^{2}} g_{\perp},
$$

where $Z_{0}=\mu_{0} c=377 \Omega, R=30.3 \mathrm{~m}$ is the average radius of the synchrotron, and $\beta$ and $\gamma$ are the standard relativistic factors, which are time-varying due to the acceleration. The longitudinal impedance is normalized by the mode numbers, $n=\omega / \omega_{0}$, where $\omega_{0}$ is the revolution frequency (1.1 to $1.5 \mathrm{MHz}$ ). 


\section{DISCLAIMER}

This report was prepared as an account of work sponsored by an agency of the United States Government. Neither the United States Government nor any agency thereof, nor any of their employees, make any warranty, express or implied, or assumes any legal liability or responsibility for the accuracy, completeness, or usefulness of any information, apparatus, product, or process disclosed, or represents that its use would not infringe privately owned rights. Reference herein to any specific commercial product, process, or service by trade name, trademark, manufacturer, or otherwise does not necessarily constitute or imply its endorsement, recommendation, or favoring by the United States Government or any agency thereof. The views and opinions of authors expressed herein do not necessarily state or reflect those of the United States Government or any agency thereof. 


\section{DISCLAIMER}

Portions of this document may be illegible in electronic image products. Images are produced from the best available original document. 
The ceramic vacuum chamber is constructed with a special if shield, shown in Figure 1, to minimize both the space charge coupling impedance and eddy currents. The shield consists of axially-mounted $\mathrm{Be}-\mathrm{Cu}$ wires which follow the beam envelope at an aperture equal to the beamstay-clear (BSC). The beam has an elliptical cross-section, its major axis alternating between the horizontal and vertical planes. However, in the standard approximation, the geometrical factors, $g_{0}$ and $g_{\perp}$, are evaluated assuming a uniform, round, unbunched beam of radius $a$ in a vacuum chamber of radius $b: 1,2,4$

$$
g_{0}=\alpha+2 \ln \left(\frac{b}{a}\right) \quad g_{\perp}=\frac{1}{a^{2}}-\frac{1}{b^{2}}
$$

The transverse beam radius, $a$, decreases by a factor of 1.8 during the acceleration cycle ( 400 $\mathrm{MeV}$ to $2 \mathrm{GeV}$ ). The parameter $\alpha$ varies parabolically from unity on axis to zero at the beam edge. 1 The average over a uniform beam gives 0.5 . However, an argument is made that for emittancedominated beams, a constant-radius model is appropriate, in which case $\alpha=1.4$

The impedance is estimated by using the average beam and $\mathrm{rf}$ shield radii, which are $50 \mathrm{~mm}$ and $70 \mathrm{~mm}$, respectively, at injection. The if shield is modelled as a smooth round beam pipe. The difference between calculating $Z_{\|}$using the average values of $a$ and $b$, and calculating the average value of $Z_{\|}$directly, is no more than $1 \%$ for the longitudinal and $10 \%$ for the transverse. The results for the space charge impedance during the acceleration cycle are shown in Figure 2 for both the longitudinal and transverse planes. As shown in Table 1, compared with a fixed-radius rf shield $\left(111 \mathrm{~mm}\right.$ at the maximum lattice function, $\beta_{\mathrm{x}}$ ), this contour-following scheme reduces the longitudinal space charge impedance by $30 \%$ at injection ( $400 \mathrm{MeV}$ ) and $20 \%$ at extraction (2 $\mathrm{GeV}$ ). The transverse impedance is reduced by $35 \%$ at injection and $10 \%$ at extraction.

Table 1. Space Charge Impedance Estimation for the RCS

\begin{tabular}{lccc}
\hline & $\begin{array}{c}\text { contour- } \\
\text { following rf } \\
\text { shield }\end{array}$ & $\begin{array}{c}\text { fixed-radius } \\
\text { rf shield }\end{array}$ & units \\
\hline $\begin{array}{l}\text { longitudinal: injection } \\
\text { longitudinal: extraction }\end{array}$ & 220 & 330 & $\Omega$ \\
transverse: injection & 50 & 65 & $\Omega$ \\
transverse: extraction & 2.8 & 4.3 & $\mathrm{M} \Omega / \mathrm{m}$ \\
\hline
\end{tabular}




\section{Wire RF Shield}

The electrostatic fields due to a uniform beam propagating inside a round (fixed-radius) rfscreening wire cage have been derived by $\mathrm{T}$. Wang. 5 The impedance is modified by the following approximate expressions for the geometrical factors (no exterior conductiong beam pipe):

$$
g_{0}=1+2 \ln \left(\frac{b}{a}\right)+F_{1} \quad g_{1}=\frac{1}{a^{2}}-\frac{F_{2}}{b^{2}}
$$

The parameters $F_{1,2}$ are given by

$$
F_{1}=\frac{1}{\frac{1}{\ln (n b)}+\frac{N}{\ln \left(8 \pi^{2} n r_{W} / N^{2}\right)}} \quad F_{2}=\frac{N}{N \ln (n b)+\ln \left(8 \pi^{2} n r_{W} / N^{2}\right)}
$$

where $N$ is the number if evenly-spaced wires of radius $r_{W}, b$ is the rf shield radius, and $n$ is the wave number of the perturbation. For the longitudinal case, the wave number of interest corresponds to the mode numbers $n \leq 500$, using the cutoff frequency $\omega_{c}=c / b$. For the transverse case, $\omega=(p+v) \omega_{0}$, where $p$ is a negative integer near $v$, the horizontal or vertical tune $(n \leq 1)$.

\section{Elliptical Beam Cross Section}

A second correction takes into account the varying elliptical shape of the beam. A smooth, metalized vacuum chamber is assumed without wires and with a fixed radius. The geometrical factors, derived by H. Okamoto 6 , are written

$$
g_{0}=1+2 \ln \left(\frac{2 b}{a_{x}+a_{y}}\right) \quad g_{\perp x, y}=\frac{2}{a_{x, y}\left(a_{x}+a_{y}\right)}-\frac{1}{b^{2}} .
$$

where $a_{x}$ and $a_{y}$ are the horizontal and vertical beam half-axes, respectively. In the longitudinal plane, this correction reduces the fluctuation in the impedance and, likewise, the space charge potential, over the circumference of the ring. In the transverse plane, the correction is significant if the $x, y$ beam sizes differ greatly.

\section{Comparison}

The impedance using the standard $g$ factors is now compared to the formulae which include corrections due to the wires and the elliptical beam shape variation. Since the corrections are both derived for a fixed-radius if shield or beam pipe, the comparison is likewise made with a fixed- 
radius rf shield in the standard, uniform, round beam assumption. The wire radius is assumed to be $0.75 \mathrm{~mm}$, as in the ISIS design 7 , and the wires are placed on a fixed radius of $111 \mathrm{~mm}$ (153 wires) with a $33 \%$ fill ratio. A comparison at $400 \mathrm{MeV}$ is given in Table 2 , where $F_{1}=-0.09$ and $F_{2}=0.20$. These corrections result in a difference of less than $2 \%$ in the longitudinal impedance. Wang's method results in a $+15 \%$ correction in the transverse impedance and Okamoto's method results in a $-20 \%$ correction. Until a rigorous derivation is performed for a contour-following wire If shield, the results using the standard $g$ factors are used for the stability analysis, and the above corrections are treated as an uncertainty.

Table 2. Comparison of the standard space charge impedance with the corrections $(400 \mathrm{MeV})$

\begin{tabular}{ccccc}
\hline & beam model & rf shield wall & $\begin{array}{c}\left|\mathrm{Z}_{\mid} / \mathrm{n}\right| \\
(\Omega)\end{array}$ & $\begin{array}{c}\left|\mathrm{Z}_{\perp}\right| \\
(\mathrm{M} \Omega / \mathrm{m})\end{array}$ \\
\hline standard & uniform, round & smooth & 335 & 4.3 \\
wires & uniform, round & wires & 326 & 5.3 \\
elliptical & uniform, elliptical & smooth & 337 & 3.6 \\
\hline
\end{tabular}

The author would like to thank K. Thompson for supplying Figure 1.

\section{References}

1. V.K. Neil and A.M. Sessler, "Longitudinal Resistive Instabilities of Intense Coasting Beams in Particle Accelerators," Rev. Sci. Instrum. 36(4), 429 (1965).

2. L.J. Laslett, V.K. Neil and A.M. Sessler, "Transverse Resistive Instabilities of Intense Coasting Beams in Particle Accelerators," Rev. Sci. Instrum., 36(4), 436 (1965).

3. K.-Y. Ng, "Fields, Impedances, and Structures," in The Physics of Particle Accelerators, AIP Conf. Proc. 184, p. $515 f$ (1989).

4. J. G. Wang, H. Suk, D. X. Wang, and M. Reiser, "Determination of the Geometry Factor for Longitudinal Perturbations in Space-Charge Dominated Beams," Phys. Rev. Letters, 72, 13, 28 Mar 1994.

5. T-S. F. Wang, Electrostatic Field of a Perturbed Beam with RF-Screening Wires, CERN Report No. PS 94-08 (DI) (1994).

6. AUSTRON Accelerator Feasibility Study, Chapter 4, p. 145 (Oct. 26, 1994).

7. KAON Factory Study, Accelerator Design Report, p. 6-7. 


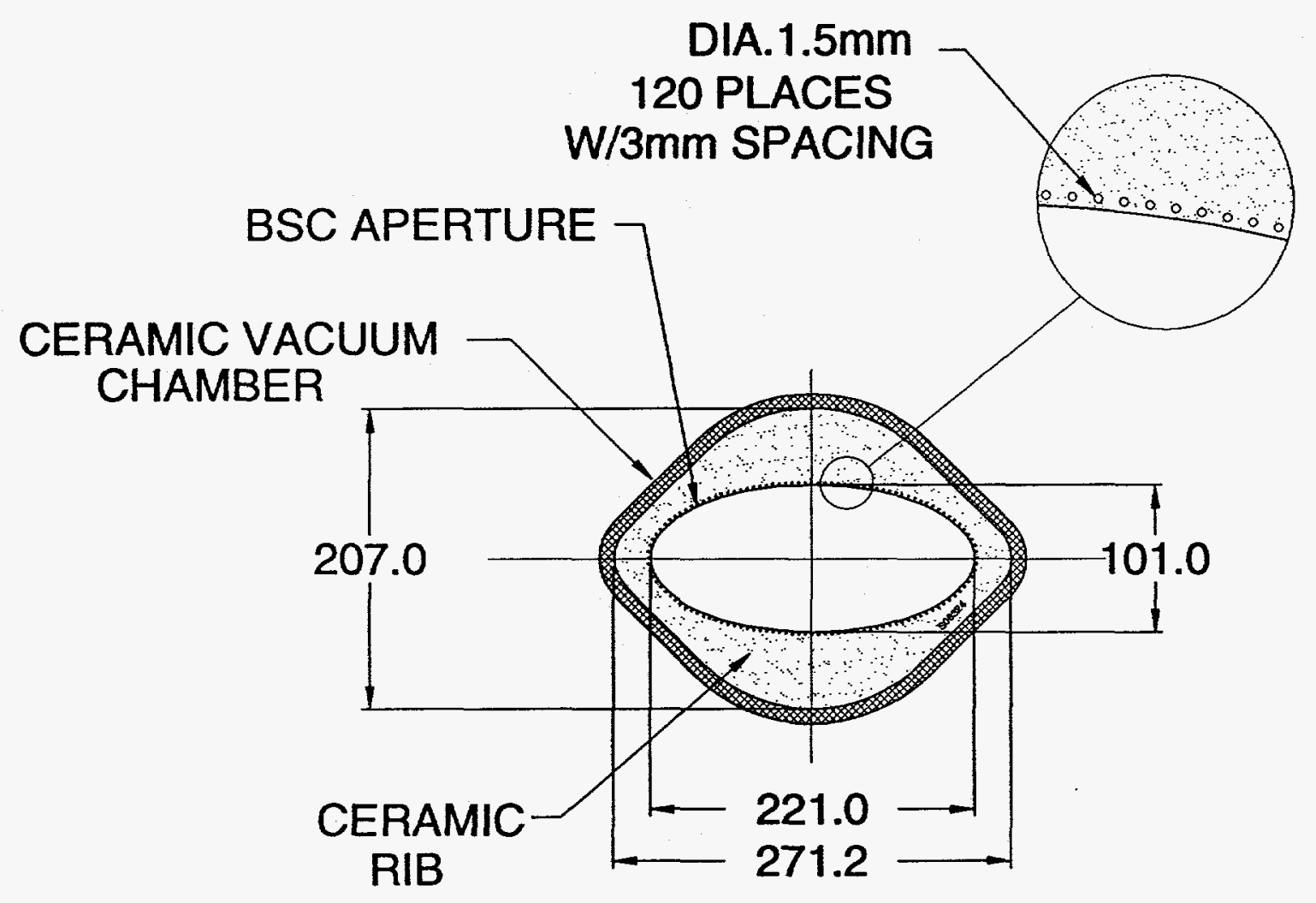

Figure 1: Ceramic vacuum chamber cross section in the focusing quadrupole magnet showing rf-screening wire shield. 

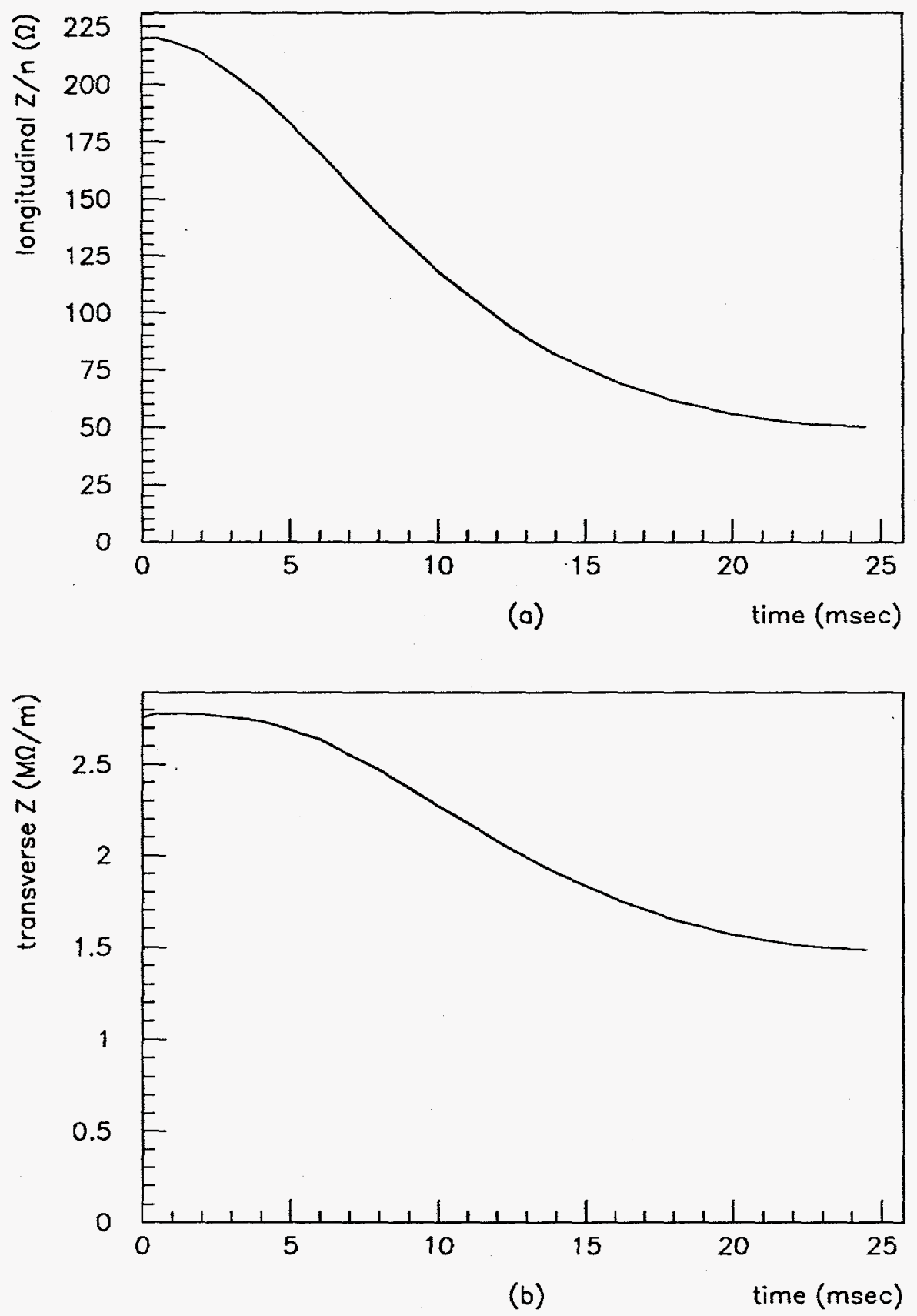

Figure 2: Time variation of (a) longitudinal and (b) transverse space charge impedance in the RCS using a contour-following wire if shield and the standard geometrical factors. 